\title{
A survey of managed care strategies for pregnant smokers
}

\author{
Dianne C Barker, Lucy A Robinson, Abby C Rosenthal
}

\begin{abstract}
Objective-The purpose of this study was to measure the content and comprehensiveness of pregnancy specific smoking cessation strategies within managed care organisations (MCOs) responding affirmatively to the national 1997-98 Addressing Tobacco in Managed Care (ATMC) survey.
\end{abstract}

Design-This cross sectional follow up study consisted of a fax survey sent to medical directors and a 37 question telephone survey of program overseers about the smoking cessation strategy.

Subjects-147 MCOs identifying a pregnancy specific smoking cessation strategy on the 1997-98 ATMC survey served as the initial sample; $88 \mathrm{MCOs}$ of 128 eligible plans completed both components, with a response rate of $69 \%$.

Results-Pregnancy specific smoking cessation strategies varied. $40 \%$ of respondents used the Agency for Health Care Policy and Research guidelines for clinical smoking cessation to design their strategy. Strategies included self help materials, quit classes, telephone support and brief counselling by providers, linkages to quality improvement efforts, and use of patient databases for outreach. Only $42 \%$ offered a postpartum relapse prevention element. Lack of patient interest, competing clinic priorities, and the lack of a smoker identification system were the most problematic barriers to implementing strategies, common to at least a quarter of respondents. A majority ranked best practice manuals and web site linkages as the most useful form of technical assistance, followed by peer-to-peer counselling, regional workshops, newsletters, on-site assistance, and national conferences.

Conclusions-The survey provides the first profile of prenatal tobacco treatment strategies in managed care. While design limitations prevent generalisation of these results to all MCOs, such information can help guide technical assistance to plans interested in reducing smoking among pregnant women.

(Tobacco Control 2000;9(Suppl III):iii46-iii50)

Keywords: managed care organisation; pregnancy; smoking cessation

Smoking cessation is one of the most cost effective interventions in medicine ${ }^{1}$ and has been labelled "the gold standard" of preventive health care. ${ }^{2}$ In cost per life-year saved, smoking cessation interventions can cost a few hundred to a few thousand dollars, depending on the level of intensity, compared with $\$ 20000$ to $\$ 100000$ plus for secondary and tertiary health care procedures. ${ }^{3}$ Smoking cessation in pregnancy reaps the same if not greater benefits. For every dollar invested in smoking cessation for pregnant women, about $\$ 3$ is saved in neonatal intensive care costs and another $\$ 3$ in long term care costs associated with low birth weight deliveries. ${ }^{4}$

Most Americans receive health care services from managed care organisations (MCOs); however, insurance coverage for smoking cessation services are not widespread..$^{5-7}$ Data from 33 states indicate that pregnant women aged 18 years and over smoke less than their non-pregnant counterparts, but prevalence rates for pregnant women still range from 5\% for women with more than a high school education to a high of $23 \%$ in women with less than a high school education. ${ }^{8}$ More than two thirds $(68 \%)$ of smokers state they want to quit smoking, ${ }^{9}$ and among currently smoking women of childbearing age, $50.3 \%$ have attempted to quit smoking for at least one day in the past year. ${ }^{8}$ Physician advice to quit smoking is noted as one of the most important motivators in a smoker's decision to quit. ${ }^{10}$ Since pregnant women visit a provider about 12 times during pregnancy, ${ }^{11}$ MCOs, health care providers, and their office staff can play a unique role in smoking cessation interventions. Smoking cessation counselling is especially effective for smokers at special risk, including pregnant women, whose quit rates double when counselled. ${ }^{12}$ Providers also may intervene with highly motivated pregnant smokers who spontaneously quit when they first learn of their pregnancy but may require ongoing help to prevent relapse.

The Agency for Health Care Policy and Research (AHCPR) (renamed the Agency for Healthcare Research and Quality in December 1999) smoking cessation clinical practice guideline for health care practitioners offers major recommendations to treat tobacco dependence and promote quitting in all smokers including pregnant smokers. ${ }^{13}$ However, little is known about the extent to which these recommendations have been incorporated into health care settings either for the general population or for pregnant women.

The survey described below represents the first endeavour to quantify the extent and content of existing pregnancy specific smoking cessation initiatives offered by managed care 
plans. Such information can help guide strategic planning at health plans, grantmaking agencies, and technical assistance organisations interested in reducing smoking among pregnant women.

\section{Methods}

The Addressing Tobacco in Pregnancy Survey was conducted by the National Technical Assistance Office for Addressing Tobacco in Managed Care (NTAO), a program of the Robert Wood Johnson Foundation directed by the American Association of Health Plans (AAHP) and the Health Alliance Plan in Detroit, Michigan. The sample of MCOs originated from an initial Addressing Tobacco in Managed Care (ATMC) mail survey conducted by the AAHP and the Prudential Center for Health Care Research in 1997-98. The ATMC survey derived its sample from the AAHP database, consisting of all member and non-member plans in the USA in 1996. Of these 637 plans on the database, 95 were excluded from the sample because of duplication, mergers or closures. Three hundred and twenty three of these 542 eligible MCOs responded to the ATMC survey, resulting in a response rate of $60 \%$. Forty five per cent or 147 of the responding plans indicated having a specific smoking cessation strategy targeting pregnant women. ${ }^{14}$

These 147 plans served as the initial sample for the 1999 Addressing Tobacco in Pregnancy (ATIP) survey. Of these, eight had closed or merged by 1999, leaving 139 initially eligible for the ATIP sample. The survey had two components, implemented sequentially. The first component consisted of a nine question tobacco policy survey that was faxed to MCO medical directors. One hundred and three medical directors $(74 \%)$ responded. The fax survey queried plans about benefit structure, clinical guideline usage, and general program information. In addition, the medical director named the person directly responsible for implementing the plan's prenatal smoking cessation program, hereinafter known as the program overseer. The second component consisted of a 37 question telephone interview with the program overseers. This survey questioned program overseers about specific program features (for example, types of interventions and patient materials, provider incentives, training approaches, quality improvement efforts, patient databases), technical assistance needs, and resource allocation. Instruments for both components were reviewed by other experts in the field, and were field tested with five health plans before the full fieldwork began in April 1999.

Of the 103 medical care directors responding to the fax survey, 92 indicated that a smoking cessation strategy for pregnant smokers currently existed at their MCO. As a result of this screening, the total number of known eligible plans was reduced to 128 plans. Representatives from the 92 health plans were contacted between April and July 1999 and were asked to complete the program overseer survey. Only four program overseers refused to participate, resulting in completed interviews for $88(69 \%)$ of the eligible health plans.

\section{Results}

Of the 88 plans, $20 \%$ were group or staff model MCOs, $52 \%$ were individual practice associations (IPAs), and 25\% had both group/ staff entities as well as IPAs. Three plans did not describe their managed care model. Plan size ranged from 13000 enrollees to 15 million enrollees, with a median of 150000 members. Two plans were Medicaid-only plans, 46 plans contracted commercially and with Medicaid, three plans contracted with Medicaid but did not indicate whether they also contracted commercially, 33 were commercial only plans, and four plans reported missing data.

Respondents to the program overseer survey included health education, promotion and/or wellness directors, coordinators and/or managers $(25 \%)$, health educators $(14 \%)$, quality improvement or quality management staff $(14 \%)$, medical directors $(12 \%)$, maternal and child health personnel (9\%), administrators $(9 \%)$, nurses $(8 \%)$, and marketing personnel (2\%). The remaining $7 \%$ did not identify their role within the MCO.

GENERAL PROGRAM DESIGN AND POLICIES

Medical directors were asked to rate the level of importance of five factors in the plan's decision to develop and launch a smoking cessation strategy for pregnant women. Over three quarters $(77 \%)$ indicated that the number and cost of low birth weight babies was very or somewhat important. Other factors deemed very or somewhat important were demand from providers $(67 \%)$, demand from patients $(59 \%)$, demand from purchasers $(57 \%)$, and competition with other health plans in the market (39\%). Additionally, 12 plans (14\%) volunteered that providing smoking cessation assistance for pregnant women was simply appropriate prenatal care, and nine $(10 \%)$ said their programs were launched to improve health outcomes and reduce morbidity.

Health plans generally chose to design a specialised smoking cessation program for their pregnant population $(69 \%)$, although $31 \%$ of respondents invited their pregnant smokers to participate in the plan's tobacco treatment program open to all members. Of those 79 plans which contracted commercially, most plans $(81 \%)$ made their smoking cessation strategies available to all commercially enrolled members, $15 \%$ said eligibility depended on the individual's benefits package, and only three plans $(4 \%)$ did not provide a smoking cessation strategy for commercially enrolled pregnant women. For those 51 plans which contracted with Medicaid, $90 \%$ offered smoking cessation to Medicaid enrollees. Specific interventions varied in the amount of cost assumed by the participant. About half of the plans offering classes or telephone support covered all the costs for a commercially enrolled member who enrols in a smoking cessation class $(55 \%)$ or telephone counselling $(48 \%)$. About a third $(36 \%)$ of commercial 
Table 1 Smoking cessation strategy implementation barriers as perceived by program overseers $(\%)(n=88)$

\begin{tabular}{|c|c|c|c|c|c|}
\hline & Very problematic & $\begin{array}{l}\text { Somewhat } \\
\text { problematic }\end{array}$ & Not problematic & $\begin{array}{l}\text { Don't } \\
\text { know }\end{array}$ & Missing \\
\hline Lack of models appropriate to their setting & 15 & 24 & 53 & 3 & 5 \\
\hline Providers reluctant to counsel their patients & 13 & 43 & 39 & 1 & 4 \\
\hline Competing priorities in the prenatal clinic & 28 & 41 & 15 & 5 & 11 \\
\hline Difficulties training providers & 22 & 31 & 26 & 2 & 19 \\
\hline Lack of patient interest & 33 & 35 & 25 & 1 & 6 \\
\hline Pregnant women reluctant to admit to smoking & 18 & 41 & 30 & 7 & 5 \\
\hline No system to identify and track smokers & 28 & 22 & 44 & 0 & 6 \\
\hline Lack of a good evaluation mechanism & 24 & 34 & 35 & 1 & 6 \\
\hline Lack of organisational support & 13 & 27 & 53 & 2 & 5 \\
\hline Lack of interest from plan purchasers & 6 & 26 & 56 & 7 & 6 \\
\hline
\end{tabular}

plans providing individualised counselling covered all costs.

Only $51 \%$ of program overseers were able to estimate their member reach. Of those who did, 54\% indicated that less than a quarter of their pregnant smoker population was participating. As indicated in table 1, lack of patient interest was viewed as a major barrier to program implementation.

Forty per cent of respondents used the 1996 AHCPR guidelines to design their smoking cessation strategy while $39 \%$ did not. The remainder did not know $(17 \%)$ or did not answer (4\%). Of these 35 plans employing the AHCPR guidelines, 12 also developed and distributed their own guidelines. Another 11 plans developed their own guidelines without referring to the AHCPR guidelines. Finally, $26 \%$ of the plans (13 of these 23 plans had developed their own clinical guidelines) reported having a written policy regarding the use of nicotine replacement therapy or bupropion (Zyban; GlaxoWellcome) during pregnancy, $49 \%$ did not have a policy, and $25 \%$ did not know or did not answer.

Smoking cessation interventions that are linked to overall health plan quality improvement efforts suggest that smoking cessation is a central priority for a health plan. Half or 44 plans said their smoking cessation program was linked to quality improvement decision making teams, 38 plans $(43 \%)$ indicated that tobacco use reduction was a quality improvement target, 36 plans (41\%) gave feedback to providers regarding their interventions with pregnant smokers, 32 plans $(36 \%)$ conducted chart audits, and 10 plans $(11 \%)$ had formulated a tobacco oversight committee. Few plans offered financial incentives $(n=9)$, disincentives $(n=3)$ or public recognition/awards $(n=6)$ to encourage providers to help pregnant women quit smoking.

Patient databases can provide useful data for smoker outreach programs and evaluation. Of the 55 plans $(61 \%)$ who had an electronic patient database, $70 \%$ captured smoking status, $32 \%$ recorded whether or not a provider had advised a patient to quit, $30 \%$ included the smoking status of other household members, and $26 \%$ indicated the stage of change on the database. Only $47 \%$ of plans who captured smoking status used this information to outreach to pregnant smokers, and few plans $(\mathrm{n}=9)$ sent notices using it to alert providers to smokers in their practice. While respondents were not asked directly whether or not this patient database was used for evaluation, only $24 \%$ of all program overseers indicated that lack of a good evaluation mechanism for their strategy was very problematic (table 1 ).

Health plans varied in the amount of resources dedicated to a prenatal smoking cessation strategy. The number of staff ranged from 0.05 full time equivalent (FTE) to 20 FTEs, with a median of 1.25 FTEs. Half of the 41 plans responding spent $\$ 48000$ or less per year (including personnel) on this strategy, with a range from $\$ 500$ to $\$ 250000$.

SPECIFIC SMOKING CESSATION INTERVENTIONS Most plans offered pregnant smokers at least one of the following interventions: mailed self help materials ( $84 \%$ ); brief counselling by providers during a prenatal visit $(82 \%)$; referrals to quit smoking classes (78\%); and telephone quitting assistance $(71 \%)$. The most frequently used education materials were from the American Lung Association, American Cancer Society, March of Dimes, and local health departments. Fifteen per cent of plans used materials they developed on their own. Three fifths of plans contracted with a vendor or community based program to conduct some or all of these classes and telephone support. External class referrals were mainly to local health departments, local hospitals, the American Cancer Society programs, and the American Lung Association programs. The most popular telephone support referrals were to state help lines, the Smith Klein Beecham's Committed Quitters program, and smoking cessation telephone counselling programs run by local community organisations.

Less than half $(43 \%)$ provided individualised, intensive counselling with smoking cessation specialists, and about the same percentage $(42 \%)$ offered postpartum relapse prevention assistance. For parents who smoke, only a third (33\%) of respondents asked paediatricians to intervene, and for those 47 plans with home visiting programs, slightly more than half (55\%) included smoking cessation help.

Program overseers used a variety of training approaches to educate their prenatal practices about smoking cessation interventions. A third of respondents mailed self instruction manuals to practices, while $28 \%$ organised group discussions about smoking cessation and 26\% conducted on-site training at practices. Only 11 plans (13\%) arranged add-on smoking cessation discussions to existing seminars like grand rounds. 
Table 2 Practice adherence with AHCPR smoking cessation clinical practice guidelines as perceived by program overseers (\%) $(n=88)$

\begin{tabular}{|c|c|c|c|c|c|c|}
\hline & All & Some & Few & None & $\begin{array}{l}\text { Don't } \\
\text { know }\end{array}$ & Missing \\
\hline \multicolumn{7}{|l|}{ Use of $4 \mathrm{As}$} \\
\hline Screen (ask) for tobacco use at first visit & 57 & 36 & 1 & 0 & 3 & 2 \\
\hline Ask about smoking at every visit & 18 & 53 & 9 & 11 & 7 & 1 \\
\hline Strongly advise smokers to quit & 46 & 49 & 1 & 0 & 3 & 1 \\
\hline Briefly counsel (assist) the patient in quitting & 21 & 50 & 15 & 1 & 12 & 1 \\
\hline Arrange for follow-up support & 15 & 50 & 24 & 3 & 7 & 1 \\
\hline \multicolumn{7}{|l|}{ Use of office systems } \\
\hline Modify patient intake forms so that smoking status in requested & 48 & 25 & 3 & 1 & 11 & 11 \\
\hline Use a chart stamp or sticker to identify smokers & 9 & 25 & 16 & 19 & 21 & 10 \\
\hline Modify the forms so that tobacco use is a vital sign & 18 & 27 & 11 & 11 & 22 & 10 \\
\hline Identify a smoking cessation coordinator in their practice & 13 & 13 & 35 & 19 & 10 & 10 \\
\hline Record a nicotine dependence code & 2 & 11 & 22 & 27 & 27 & 10 \\
\hline
\end{tabular}

When asked to estimate how many prenatal practices in their plan implement the recommended AHCPR guidelines for primary care clinicians, program overseers perceive that a considerable number screen pregnant women for smoking at the first prenatal visit and strongly advise pregnant women to quit smoking (table 2). However, the extent of practices that ask about smoking status at every visit, provide brief counselling (3-10 minutes), and arrange for follow up support appear more limited. According to program overseers, as shown in table 2, many practices have yet to implement smoker identification systems. Moreover, only $26 \%$ of all respondents estimated that all or some of their prenatal practices have identified someone in their office to coordinate smoking cessation efforts.

HEALTH PLAN PERCEPTIONS OF TECHNICAL ASSISTANCE NEEDS

Respondents were asked to state their biggest barrier in implementing their smoking cessation strategy for pregnant women, and then were given a list of barriers to rate how problematic each had been. Not one barrier was prominent, although surprisingly only seven respondents mentioned resources as a barrier under the open ended question. Lack of patient interest, competing priorities in the prenatal clinic, and the lack of a system to identify and track smokers were the most troublesome barriers common to at least a quarter of all program overseers. A majority of respondents did not view lack of interest from plan purchasers, lack of organisational support or lack of an appropriate model as problematic (table 1).

Most program overseers (88\%) were interested in participating in a network of health plans to share information about prenatal smoking cessation programming. To help guide technical assistance offered by the ATMC NTAO, program overseers rated how useful a specific technical assistance approach would be to them. A majority of respondents indicated that the distribution of a best practice manual (55\%) and web site linkages (50\%) would be very useful, followed by peer-to-peer teleconferencing $(38 \%)$, regional workshops $(35 \%)$, newsletters $(33 \%)$, on-site assistance $(28 \%)$, and national conferences (26\%). Program overseers also were asked to assess whether they were most likely, somewhat likely or not likely to use specific types of technical assistance. Respondents were most enthusiastic about receiving resource materials $(66 \%$ stated most likely) followed by information about relapse prevention strategies (57\%), state-of-the-art smoking cessation interventions $(47 \%)$, organisational support strategies $(34 \%)$, provider training (33\%), and system change implementation (32\%).

\section{Discussion}

As the first profile of such strategies in managed care nationally, the results from this survey provide important baseline information to those interested in facilitating prenatal cessation services in managed care.

Design limitations, coupled with a $40 \%$ nonresponse rate from the original survey, however, prevent generalisation of these results to all managed care plans. The sampling frame was restricted to only those plans from the original ATMC survey which indicated that they had a prenatal smoking cessation strategy. Although the response rate for our ATIP survey was reasonable, the denominator excluded the original number of known eligible plans (542 plans). By definition, those not eligible for our survey did not have pregnancy specific smoking cessation strategy. Furthermore, if one assumes that non-respondents to the original ATMC survey were less likely to have general tobacco control programs, national prevalence estimates of various program features (clinical guideline use, telephone support, postpartum programs, etc) are apt to be substantially lower than those reported in our survey. As such, our survey yields the most favourable scenario likely to exist in managed care organisations; thus the need for technical assistance to design evidence based prenatal smoking cessation services is even greater than implied by survey results.

A second major limitation of this survey derives from its original design as a top down survey capturing health plan management's knowledge about program design and intent to treat. We carefully chose two categories of respondents (medical directors and program overseers) recommended by other experts in the field as the best contacts to represent the plan's perspective. While our program overseers reflected many position titles, these program overseers all were named by the medical director as directly responsible for implementing the smoking cessation strategy. Given resource limitations and anticipated 
respondent burden concerns, we purposely designed this survey to include only one program overseer respondent. The survey intentionally did not assess the actual implementation of prenatal smoking cessation strategies at the health care delivery level. Ideally, we would have liked to interview several respondents per plan and included a random sample of practices. As practice often differs from policy, little is still known about how these strategies are actualised in the clinic setting. Moreover, further research is needed to understand how these strategies differ by type of health plan. Prenatal smoking cessation strategies in staff model MCOs, for example, may be easier to implement and monitor at the clinical level than in network models where practices often simultaneously contract with a multitude of MCOs. Because a quarter of respondents were from plans with both group/ staff entities and IPAs, and respondents were not asked to categorise response by type of entity, this survey could not provide valid differences across health plan type.

In the context of these limitations, results indicate that a number of managed care organisations across the USA have invested some resources in prenatal smoking cessation programs for their pregnant smokers, recognising the value of such programs in reducing morbidity and mortality in mothers and their children. Many plans surveyed offered quit smoking classes, telephone quit assistance, and distribution of self help materials, all acceptable alternatives for pregnant smokers. Three fifths of MCOs from the survey, however, did not rely on the simple, cost effective approach advocated by the AHCPR guidelines to design their program. These guidelines recommend the active engagement of providers, including but not limiting their role to distributing patient self help materials and arranging follow up services such as classes and telephone support. The 2000 guideline recommends formally adding "assessing" a patient's willingness to quit as a fifth step to the original, simple cost effective 4 As. ${ }^{15}$ In those MCOs already practising some of these, providers must be trained to not only advise, but assess and counsel patients, as many patients may not seek other assistance. Postpartum relapse services, although greatly needed, are present in a minority of surveyed plans. All managed care organisations would benefit from help in designing and implementing office systems to support the 5As, and in developing outreach and evaluation tools using existing databases.

Survey results indicate that plans welcome a variety of technical assistance. This assistance is readily available from the Robert Wood Johnson Foundation and the American Association for Health Plans Addressing Tobacco in Managed Care National Technical Assistance Office. In addition, a simple, low cost training approach for prenatal providers developed by the Robert Wood Johnson Foundation Smoke-Free Families Program and endorsed by the Academy of Obstetricians and Gynecologists will be disseminated nationally in 2000 .

MCOs also may leverage tobacco settlement monies and collaborate with the public health community to develop strategies to encourage pregnant women to seek these cost effective services. Lack of patient interest was a major barrier named by a third of respondents. Full benefit coverage, although more likely to yield higher utilisation of these services, ${ }^{16}$ was often not available in the surveyed plans.

Managed care has a "financial self interest" in tapping these opportunities. ${ }^{17}$ In 1997-98, maternal programs ranked highest among smoking cessation strategies offered by managed care. ${ }^{14}$ Evidence based prenatal smoking services can not only improve the health of pregnant women, their future children and their families, but serve as the model for tobacco treatment services for other target populations.

The authors wish to thank Marguerite Burns, Susan J Curry, Ronald Davis, Michael Fiore, Robert Goldenberg, C Tracy Orleans, Linda Schuessler, and Danielle Skirpak Stoffer for their assistance in developing the instrument and methodology. The authors also greatly appreciate help from Regina Yeckley in her diligence and patience in preparing the instruments and contacting non-respondents, Lisa Mahoney for sample preparation, Tahniyath Baig for conducting the majority of the interviews, and Julie Masura, Alissa Kapke, and Gary Chase for their analytical support.

1 Cromwell J, Bartosch WJ, Fiore MC, et al. Costeffectiveness of the clinical practice recommendations in the AHCPR guidelines for smoking cessation. FAMA 1997;278:1759-66.

2 Eddy DM. David Eddy ranks the tests. Harvard Health Letter July 1992 (suppl):10-11.

3 Warner KE. Smoking out the incentives for tobacco control in managed care settings. Tobacco Control 1998; in manpl):S50-4 (table 1).

4 Marks JS, Koplan JP, Hogue CJR, et al. A cost-benefit/costeffectiveness analysis of smoking cessation for pregnant women. Am F Prev Med 1990;6:282-9.

5 Schauffler HH, Parkinson MD. Health insurance coverage for smoking cessation services. Health Educ $Q 1993$; 20:185-206.

6 Schauffler HH. Defining benefits and payments for smoking cessation treatments. Tobacco Control 1997;6(suppl):S81-

7 Barker DC, Orleans CT, Schauffler HH. Tobacco treatment services should be covered under Medicaid. Tobacco Control 1998;7:92-3.

8 Ebrahim SH, Floyd RL, Merritt RK, et al. Trends in pregnancy-related smoking rates in the United States, 1987-1996. ҰAMA 2000;283:361-6.

9 Centers for Disease Control and Prevention. Cigarette smoking among adults: United States, 1995. Morbid Mortal Wkly Rep 1997;46:1217-20.

10 Fiore $M$. The three minute solution for smoking cessation. Strategic Med 1998;2:21-6.

11 Ventura SJ, Martin JA, Curtin SC, et al. Report of final natality statistics, 1996. Mon Vital Statistics Rep 1996; 46(11 suppl): $98-1120$.

12 Mullen PD, Ramirez G, Groff, JY. A meta-analysis of randomized trials of prenatal smoking cessation interventions. Am $\mathcal{F}$ Obstet Gynecol 1994;171:1328-34.

13 Fiore MC, Bailey WC, Cohen SJ, et al. Smoking cessation. Clinical Practice Guideline No 18. Rockville, Maryland: US Department of Health and Human Services, Public Health Service, Agency for Health Care Policy and
Research, April 1996. (AHCPR Publication No 960692.); <http://text.nlm.nih.gov>.

14 McPhillips-Tangum C. Results from the first annual survey on Addressing Tobacco in Managed Care. Tobacco Control 1998;7(suppl):S11-13.

15 Fiore MC, Bailey WC, Cohen SJ. Treating tobacco use and dependence. A clinical practice guideline. Rockville, Maryland: US Department of Health and Human Services, Public Health Service. June 2000.

16 Curry, SJ, Grothaus LC, McAfee T, et al. Use and cost effectiveness of smoking-cessation services under four insurance plans in a health maintenance organization. $N$ Engl f Med 1998;339:673-9.

17 Wall M. Pre- and postnatal smoking intervention in managed care settings. Tobacco Control 2000;9(suppl I):i63. 